\title{
Quantum-inspired interferometry with chirped laser pulses
}

\author{
R. KALTENBAEK*, J. LAVOIE, D. N. BIGGERSTAFF AND K. J. RESCH* \\ Institute for Quantum Computing and Department of Physics and Astronomy, University of Waterloo, Waterloo, N2L 3G1, Canada \\ *e-mail: rkaltenb@iqc.ca; kresch@iqc.ca
}

Published online: 12 0ctober 2008; doi:10.1038/nphys1093

Interference is a defining feature of both quantum and classical theories of light, enabling the most precise measurements of a wide range of physical quantities including length ${ }^{1}$ and time $^{2}$. Quantum metrology exploits fundamental differences between these theories for new measurement techniques and enhanced precision ${ }^{3,4}$. Advantages stem from several phenomena associated with quantum interferometers, including non-local interference ${ }^{5,6}$, phase-insensitive interference ${ }^{7}$, phase super-resolution and super-sensitivity ${ }^{8-10}$, and automatic dispersion cancellation ${ }^{6,11,12}$. However, quantum interferometers require entangled states that are in practice difficult to create, manipulate and detect, especially compared with robust, intense classical states. In the present work, we report an interferometer based on chirped femtosecond laser pulses and classical nonlinear optics showing all of the metrological advantages of the quantum Hong-Ou-Mandel interferometer ${ }^{7}$, but with 10 million times more signal. Our work emphasizes the importance of delineating truly quantum effects from those with classical analogues ${ }^{10,13,14}$, and shows how insights gained from quantum mechanics can inspire novel classical technologies.

Arguably the best known example of quantum interference was demonstrated by Hong, Ou and $\mathrm{Mandel}^{7}$ (HOM); their interferometer is depicted in Fig. 1a. HOM interference is central to optical quantum technologies, including quantum teleportation ${ }^{15}$ and linear-optical quantum computing ${ }^{16}$. Several characteristics distinguish HOM from classical interference, such as Michelson's or Young's. The HOM signal stems from pairs of interfering photons and is manifest as a dip in the rate of coincident photon detections spanning the coherence length of the light, as opposed to classical wavelength fringes. It is therefore inherently robust against path-length fluctuations. If the photons are entangled, the visibility and width of the HOM interferogram are insensitive to loss ${ }^{17}$ and dispersion ${ }^{11}$. Furthermore, HOM interferometers achieve higher resolution than classical interferometers using the same bandwidth ${ }^{18,19}$. These features are ideal for precision optical path measurements of dispersive and lossy materials, implemented by placing the sample in one interferometer arm and measuring the delay required to restore interference. A quantum version of optical coherence tomography ${ }^{20}$ was proposed and demonstrated ${ }^{18,19}$ to harness these advantages.

Recently, two proposals ${ }^{21,22}$ and one experimental demonstration $^{23}$ have described classical systems showing automatic dispersion cancellation. Drawbacks to these techniques include reliance on unavailable technology ${ }^{21}$ or significant postprocessing ${ }^{22,23}$. The experimentally demonstrated technique requires wavelength path stability; the interference visibility falls precipitously with loss and is limited to $50 \%$ of that possible with the HOM effect. Alternatively, background-free autocorrelation of transform-limited pulses, recently used for optical coherence tomography ${ }^{24}$, shows enhanced resolution, phase insensitivity and robustness against loss, but notably not automatic dispersion cancellation.

In the present work, we describe and experimentally demonstrate classical interference with all of the metrological advantages of the HOM interferometer. Our interferometer shows a high-visibility ( $>85 \%$ measured) phase-insensitive interference dip in optical beam intensity, incorporating enhanced resolution, robustness against loss and automatic dispersion cancellation. In contrast with other classical interferometers, the Feynman paths contributing to the interference cannot be identified with the spatial paths constituting the two interferometer arms. Sum-frequency generation (SFG) directly produces the interference signal from a pair of oppositely chirped optical pulses; no postprocessing or coincidence counting is required. The device can be understood as a time-reversed HOM interferometer using an argument ${ }^{10}$ on the basis of the corresponding symmetry of quantum mechanics. Remarkably, time reversal converts the quantum interferometer into a device that can use bright classical laser pulses and achieves a demonstrated ten-million-fold higher signal. Our general approach should yield similar improvements in performance when applied to many other entangled-photon interferometers.

HOM-based metrology can be explained in the following way ${ }^{11}$. The wavevector of light in a material can be expanded about a frequency $\omega_{0}, k(\omega)=k\left(\omega_{0}\right)+\alpha\left(\omega-\omega_{0}\right)+\beta\left(\omega-\omega_{0}\right)^{2}+\cdots$, where $\alpha$ and $\beta$ are material properties describing the group delay and quadratic group velocity dispersion, respectively. Ideal frequency-entangled photon pairs are described by the state $|\psi\rangle=\int \mathrm{d} \Omega f(\Omega)\left|\omega_{0}+\Omega\right\rangle\left|\omega_{0}-\Omega\right\rangle$, where $f(\Omega)$ is the amplitude spectrum. The coincidence rate in the HOM interferometer as a function of the relative delay time, $\tau$, is given by ${ }^{11}$

$$
C(\tau)=\int \mathrm{d} \Omega|f(\Omega)|^{2}\left\{1-\cos \left[\phi_{r r}(\Omega, \tau)-\phi_{t t}(\Omega, \tau)\right]\right\} .
$$

Here $\phi_{r r}(\Omega, \tau) \quad\left(\phi_{t t}(\Omega, \tau)\right)$ are the phases associated with the amplitude where both photons are reflected (transmitted); the delay time $\tau=\left(L_{2}-L_{1}+L\right) / c$, where $L_{1}\left(L_{2}\right)$ is the length of the sample (delay) arm and $L$ is the length of the sample, and $\phi_{r r}(\Omega, \tau)=L\left(+\alpha \Omega+\beta \Omega^{2}\right)-\Omega \tau$ and $\phi_{t t}(\Omega, \tau)=L\left(-\alpha \Omega+\beta \Omega^{2}\right)+\Omega \tau$, after removing an irrelevant global phase.

Because $\phi_{r r}(\Omega, \tau)$ and $\phi_{t t}(\Omega, \tau)$ have the same dependence on the group velocity dispersion, $\beta$, it is automatically cancelled 


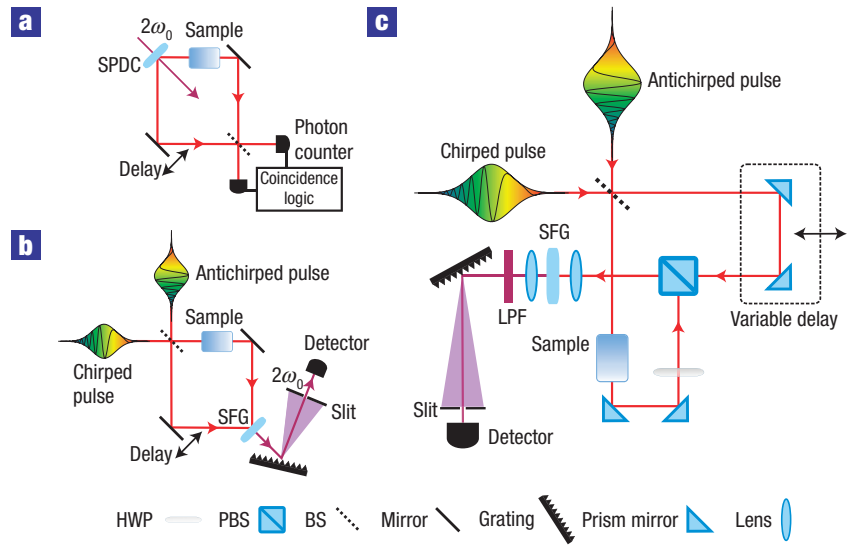

Figure 1 Chirped-pulse interferometry. a, The HOM interferometer. A laser of frequency $2 \omega_{0}$ creates frequency-entangled photons through spontaneous parametric down-conversion (SPDC). The photons propagate along different paths and are recombined at a beam-splitter (BS) after one passes through a sample. The photons can arrive at different detectors when the path lengths are unbalanced; when balance is achieved, the photons always arrive at the same detector owing to quantum interference. $\mathbf{b}$, Schematic chirped-pulse interferometer as a time-reversed HOM interferometer. Oppositely chirped laser pulses are combined at a beam-splitter. The output beams are recombined and focused onto a nonlinear crystal after one of the beams has passed through a sample. SFG near the frequency $2 \omega_{0}$ as a function of delay is detected using a standard photodiode after passing through a low-pass filter (LPF). c, Experimental set-up. The polarization is rotated in the sample path from horizontal to vertical and collinear type-II SFG is used.

in the interference signal, as are all even orders of dispersion. The coincidence rate drops to zero for $\tau=\alpha L$, or when the group delay from the material is exactly compensated by unequal physical path lengths; this marks the centre of the HOM dip.

To understand our chirped-pulse interferometry (CPI) technique, consider the cross-correlator shown in Fig. $1 \mathrm{~b}$ as a time-reversed HOM interferometer (Fig. 1a). The detection of a pair of photons with frequencies $\omega_{0} \pm \Omega$ is replaced by the preparation of a pair of photons with these frequencies; the preparation of a pump photon of frequency $2 \omega_{0}$, which is subsequently down-converted, is replaced by the detection of a photon of frequency $2 \omega_{0}$, which has previously been up-converted. The signal in equation (1) is built up by repeating the experiment with many pairs of photons with frequencies distributed according to the spectrum $|f(\Omega)|^{2}$.

The power of CPI stems from the replacement of photon pairs by bright classical beams with frequencies $\omega_{0} \pm \Omega$. The SFG from these beams will contain three distinct frequencies, instead of just one: the cross-correlation produces up-converted light at $2 \omega_{0}$ at a rate proportional to $\left\{1-\cos \left[\phi_{r r}(\Omega, \tau)-\phi_{t t}(\Omega, \tau)\right]\right\}$ (see equation (1)); the autocorrelation produces two new beams at frequencies $2 \omega_{0} \pm 2 \Omega$. A narrow bandpass filter centred at $2 \omega_{0}$ removes the autocorrelation unless $\Omega$ is small.

The frequency difference, $\Omega$, is swept using pairs of oppositely chirped optical pulses with matched frequency ramps. A chirped (antichirped) pulse has a frequency that increases (decreases) linearly in time. We require that the chirp and antichirp are much greater than any dispersion in the interferometer and stretch the pulses to many times their initial duration. Under these conditions, the frequencies are swept in an anticorrelated manner such that at any instant only two frequencies, $\omega_{0} \pm \Omega$, are input. (Oppositely chirped pulses have previously been used to efficiently

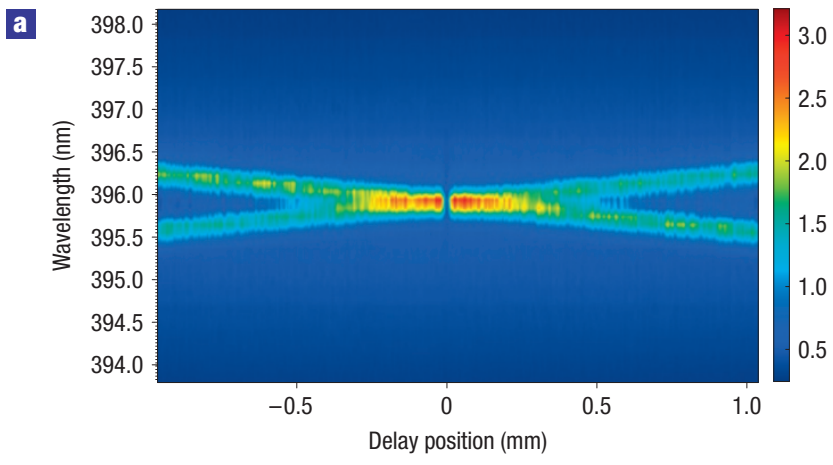

b

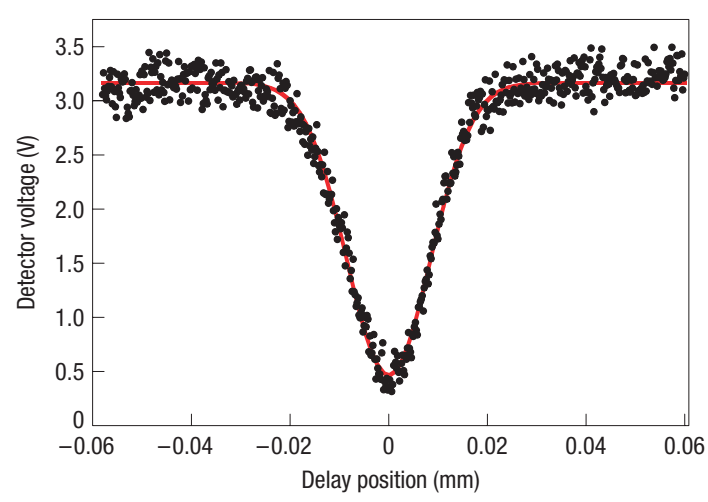

Figure 2 Chirped-pulse interference. a, Spectrum of the SFG versus delay position. Destructive interference removes the cross-correlation signal near zero delay; other features are discussed in the text. b. Using a grating and a slit, we measure the optical power at $395.9 \mathrm{~nm}$ with a bandwidth of $0.4 \mathrm{~nm}$, as a function of delay. The signal shows a pronounced dip near zero delay; we use a Gaussian fit to measure the visibility $85.2 \pm 0.6 \%$ and width $19.9 \pm 0.2 \mu \mathrm{m}$ FWHM.

drive rotational and vibrational transitions in molecules ${ }^{25,26}$.) This ramp carries out the integration in equation (1) automatically. As an added benefit, chirped pulses can have high peak intensities, yielding efficient frequency conversion.

We create beams of chirped and antichirped laser pulses ${ }^{27}$ from a mode-locked laser and inject them into the experimental set-up shown in Fig. 1c (for details, see the Methods section). The relevant centre frequency for our experiment is not the centre frequency of the pulse, but rather is determined by the temporal overlap of the chirped and antichirped pulses at the 50/50 beam-splitter. If the chirped pulse lags (leads) the antichirped pulse, the frequency $2 \omega_{0}$ is redshifted (blueshifted) from twice the centre frequency of the laser. This can be used to make measurements of group delays over a tunable range of wavelengths, which is difficult to do using HOM interference, because the entangled photons are typically produced using a fixed-frequency continuous-wave laser ${ }^{11,19}$. To illustrate this point, we combined our pulses such that the sum of the frequencies corresponded to a wavelength, $395.9 \mathrm{~nm}$, well separated from half of the centre wavelength of the laser, $395.0 \mathrm{~nm}$.

Figure 2a shows the measured SFG spectrum versus delay. The cross-correlation signal is clearly observed, but the autocorrelation signal comprises a broad background barely visible on this scale. For large delays, the cross-correlation contains two easily discernible wavelengths spaced symmetrically about $395.9 \mathrm{~nm}$. These peaks arise from SFG of the chirped component in the sample arm and the antichirped component in the delay arm, and vice versa. These different alternatives constitute the distributed 

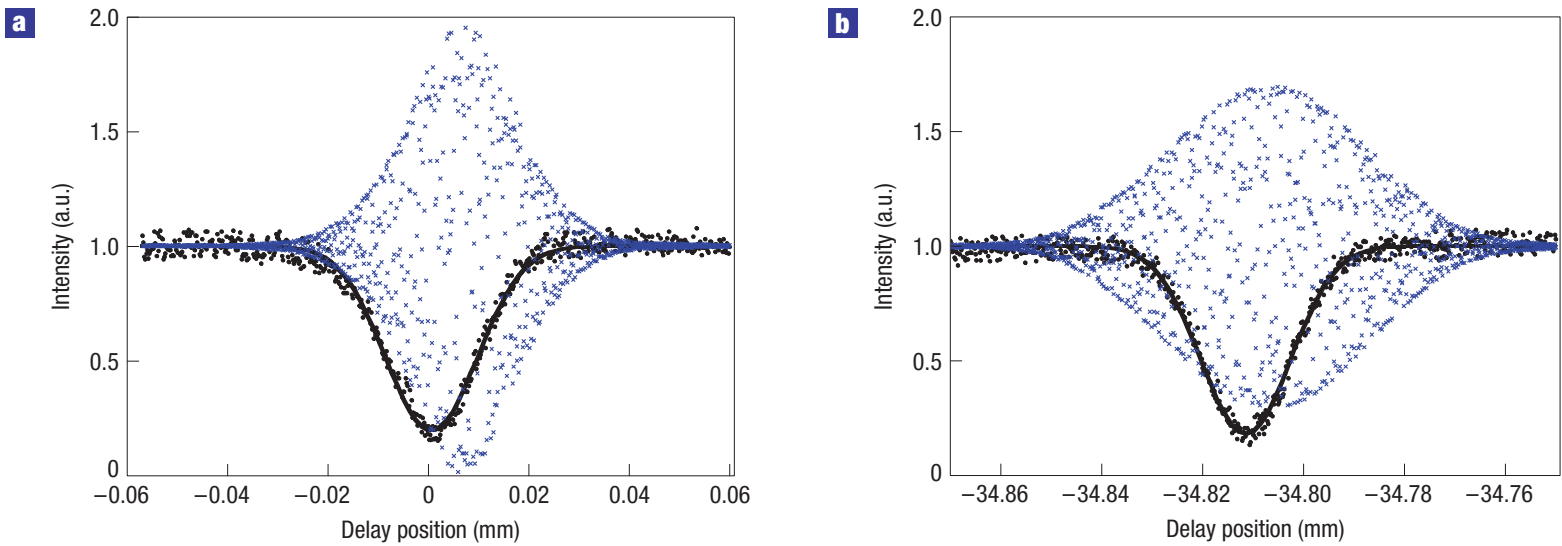

Figure 3 Automatic dispersion cancellation in chirped-pulse interferometry. a,b, A direct comparison of the chirped-pulse interference signal (black circles) and standard white-light interference using the chirped pulse (blue crosses) when no additional glass (a) and $80.60 \pm 0.05 \mathrm{~mm}$ of calcite (oriented for 0-polarization) and $28.93 \pm 0.04 \mathrm{~mm}$ of BK7 glass (b) are placed in the sample arm. These data show that the white-light interferogram is broadened by $75 \%$ whereas the $\mathrm{CPI}$ interferogram shows no broadening. Even in the case of no dispersion, the CPI interferogram has $17 \%$ better resolution. Note that the small offset between the CPI and white-light interference is due to the birefringence of the up-conversion crystal.

Feynman paths, which interfere. The two wavelengths approach one another as the path length difference approaches zero, where destructive interference eliminates the cross-correlation signal.

We filter a bandwidth of $0.4 \mathrm{~nm}$ centred at $395.9 \mathrm{~nm}$ and directly measure the intensity with an amplified silicon photodiode. The photodiode signal as a function of delay is shown in Fig. $2 \mathrm{~b}$ and clearly shows the interference dip with visibility $85.2 \pm 0.6 \%$ and full-width at half-maximum (FWHM) $19.9 \pm 0.6 \mu \mathrm{m}$ or $133 \pm 1 \mathrm{fs}$. The signal-to-noise ratio for CPI is limited by interference of the beams at the input beam-splitter; this noise source can be removed by path-length stabilization or by blocking a narrow band of frequencies near $\omega_{0}$.

Our measured visibility easily surpasses the 50\% limit commonly attributed to any classical analogue of HOM interference. This classical limit applies only to the visibility of the coincidence rate (or correlation) between two squarelaw photodetector signals showing no individual interference ${ }^{7,28}$. Although both SFG and coincidence detection measure correlations, the SFG signal depends on the product of the electric fields, as opposed to intensities; thus our detection scheme avoids this constraint. In practice, background from the autocorrelation limits the visibility, but it can be arbitrarily close to $100 \%$ with large chirp and narrow filtering. Alternatively, we could achieve $100 \%$ visibility by removing the small band of frequencies responsible for the autocorrelation background from the chirped and antichirped pulses; this has the drawback of distorting the interferogram.

The optical power corresponding to $1 \mathrm{~V}$ on our detector was measured to be $1.5 \mu \mathrm{W}$ at $395 \mathrm{~nm}$, thus our measured signal

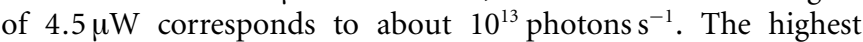
reported coincidence rate ${ }^{29}$ from a photon pair source is $2 \times 10^{6} \mathrm{~Hz}$, whereas the rate is typically orders of magnitude lower in a HOM interferometer. We demonstrate a seven-order-of-magnitude increase in signal over a HOM interferometer using state-of-the-art photon-pair sources.

To demonstrate automatic dispersion cancellation, we took two data sets: one with significant dispersive material in the sample arm, $80.60 \pm 0.05 \mathrm{~mm}$ of calcite and $28.93 \pm 0.04 \mathrm{~mm}$ of BK7 glass, and one without. In each configuration we measured chirped-pulse and white-light interferograms. To observe white-light interference, we sent the chirped pulse through the interferometer, placed a polarizer at $45^{\circ}$ before the nonlinear crystal and directly detected the transmitted infrared light. The resulting interferograms are shown in Fig. 3.

With no sample, Fig. 3a, we observe $143 \pm 2$ fs FWHM for the chirped-pulse dip and $173 \pm 1 \mathrm{fs}$ FWHM for the white-light interference pattern; the chirped-pulse signal has $17 \%$ better resolution. Integrating equation (1) with Gaussian spectra for both the chirped and antichirped pulses, that is, $|f(\Omega)|^{2}=G(\Omega) G(-\Omega)$, and comparing this to white-light interference with the spectrum, $G(\Omega)$ (equations (2)-(4) in ref. 18 ), predicts CPI to have $29 \%$ better resolution. We attribute the difference between experiment and theory mainly to lost bandwidth in SFG. With the dispersive elements, Fig. 3b, we observed $140 \pm 2 \mathrm{fs}$ FWHM for chirped-pulse interference and $303 \pm 2$ fs FWHM for white-light interference. Dispersion clearly increased the width of the white-light interference pattern by $75 \%$; the width of the CPI pattern remained essentially unchanged owing to dispersion cancellation.

To show that CPI accurately determines group delays, we measured shifts in the centre of the interference of $34,811.9 \pm 0.3 \mu \mathrm{m}$ and $34,813.80 \pm 0.3 \mu \mathrm{m}$ for the chirpedpulse dip and white-light fringes, respectively. These agree with theoretical shifts of $34,816 \pm 20 \mu \mathrm{m}$ and $34,822 \pm 20 \mu \mathrm{m}$, calculated from the group delays at $791.8 \mathrm{~nm}$ and $790 \mathrm{~nm}$, respectively. Uncertainties in the theory result from errors in the measurement of sample thickness.

A further advantage of CPI over white-light interferometry is the insensitivity of the visibility to unbalanced loss in the interferometer arms; loss will, however, reduce the overall output intensity, and thus the signal, in both cases. We measured visibilities as a function of attenuation. Rotating a half-wave plate in the sample path enables continuous adjustment of the loss at the polarizing beam-splitter. The results of these measurements are shown in Fig. 4. The visibility in the chirped-pulse interference is far more robust than that in the white-light interference, dropping only slightly at high attenuation owing solely to background. This insensitivity can be explained by noting that in CPI the loss is common to the two interfering Feynman paths even though it is localized in one physical path.

CPI features all of the metrological advantages of HOM interference with vastly higher signal levels. CPI achieves this 


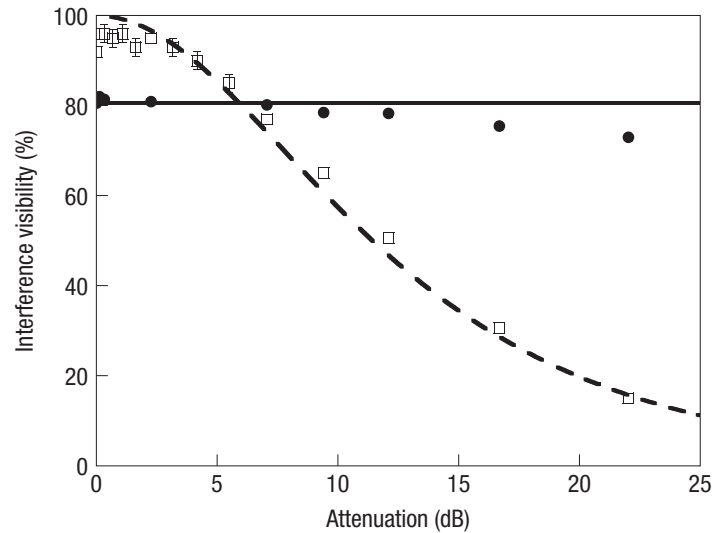

Figure 4 Visibility versus unbalanced loss. The visibility of the chirped-pulse interference dip (closed circles; solid line, theory) and white-light interference (open squares; dashed line, theory) as a function of loss in the sample arm introduced by rotating the half-wave plate (Fig. 1b). The chirped-pulse interference visibility shows far more resilience to loss. Error bars indicate statistical errors of 1 s.d. and are not shown if smaller than the data point.

without the inherent disadvantages of entangled photon sources and single-photon detection. Increasing the laser bandwidth and the spectral acceptance of $\mathrm{SFG}^{30}$ will make CPI resolution competitive with that in optical coherence tomography ${ }^{19,31}$. Automatic dispersion cancellation and insensitivity to loss and path-length fluctuations promise to make CPI a superior imaging technology, especially for dispersive and lossy media, for example biological specimens and photonic devices. This approach provides an avenue into previously untapped potential of classical interferometry.

\section{METHODS}

We use a mode-locked Ti:sapphire laser (centre wavelength $790 \mathrm{~nm}$, pulse duration $110 \mathrm{fs}$, average power $2.8 \mathrm{~W}$, repetition rate $80 \mathrm{MHz}$ ) as the light source for the experiment. The polarization of the output is rotated from vertical to horizontal using a half-wave plate to achieve maximum diffraction efficiency from our gratings. Our laser light is split using a 50/50 beam-splitter. Half of the optical power is sent through a grating-based optical compressor and the other half is sent through a grating-based optical stretcher. The stretcher applies normal dispersion, creating a chirped pulse where the blue lags the red in time, whereas the compressor applies anomalous dispersion, creating the antichirped pulse where the red lags the blue. Although the terms stretcher and compressor are commonly used, in our experiment both devices stretch our optical pulses. Both stretcher and compressor use $30 \mathrm{~mm} \times 30 \mathrm{~mm}, 1,200$ lines $\mathrm{mm}^{-1}$ gold-coated ruled diffraction gratings, blazed for $800 \mathrm{~nm}$.

In the compressor ${ }^{27}$, the gratings are oriented with their faces parallel and separated by a distance of $56 \mathrm{~cm}$. The input beam passes over the top of a prism mirror; the retro-reflecting mirror is angled slightly downward so that the output beam is reflected by the prism mirror. The compressor produces antichirped output pulses $45.1 \pm 0.1 \mathrm{ps}$ long with $9 \mathrm{~nm}$ bandwidth and the beam has an average power of $790 \mathrm{~mW}$.

In the stretcher ${ }^{27}$, the gratings are oriented with their faces antiparallel and separated by $145 \mathrm{~cm}$. A 1:1 telescope is placed between the gratings and consists of two lenses, $f \approx 50 \mathrm{~cm}$, separated by $98.5 \mathrm{~cm}$, with the first lens placed $9.2 \mathrm{~cm}$ after the first grating. The stretcher produces chirped output pulses $51.2 \pm 0.2 \mathrm{ps}$ long with $10 \mathrm{~nm}$ bandwidth and the beam has an average power of $870 \mathrm{~mW}$.

Initially, we balanced the stretcher and compressor by sending the output of the stretcher through the compressor and minimizing the pulse duration of the output by changing the grating separation in the compressor. We observed a minimum broadening of $10 \%$ over pulses directly from the laser. The differences between the durations of the chirped and antichirped pulses are due to unequal loss of bandwidth in the stretcher and compressor. They do not reflect different chirp rates.

The chirped and antichirped pulses are spatially and temporally overlapped at the input broadband beam-splitter cube of the cross-correlator shown in Fig. 1b. To compensate the shorter optical path in the compressor as compared to the stretcher, the antichirped pulse arrives at the beam-splitter via a variable delay path (not shown). We set the delay such that the sum of the frequencies of the chirped and antichirped pulses at any given time corresponded to a wavelength of $395.9 \mathrm{~nm}$. Note that this is not exactly half the centre wavelength of our laser, and can be continuously tuned by varying the time delay between chirped and antichirped input beams.

The two outputs from the beam-splitter travel different paths through the cross-correlator. One travels through the delay arm, where a retro-reflector is placed on a motorized translation stage with $40 \mathrm{~mm}$ travel; the other passes through the sample and an achromatic half-wave plate, which rotates the polarization from horizontal to vertical. The two beams are recombined at a broadband polarizing beam-splitter cube.

The output from the polarizing beam-splitter is focused by a $5 \mathrm{~cm}$ achromatic lens into a $0.5 \mathrm{~mm} \beta$-barium borate optical crystal cut for collinear type-II degenerate SFG. The sum-frequency beam is then collimated by means of another $5 \mathrm{~cm}$ lens. The infrared light is filtered by means of two dichroic mirrors (not shown) designed to reflect $395 \mathrm{~nm}$ light at $45^{\circ}$ incidence and to transmit $790 \mathrm{~nm}$ light, as well as a cyan-coloured glass low-pass filter; this is depicted as a low-pass filter (LPF) in Fig. 1b. A $0.4 \mathrm{~nm}$ band of light centred at $395.9 \mathrm{~nm}$ is filtered using a 1,200 lines $\mathrm{mm}^{-1}$ aluminium-coated diffraction grating followed by a slit. The optical power is measured using an amplified silicon photodiode (Thorlabs PDA36A). All visibilities were calculated without background subtraction; however, our photodiode registered a bias ranging from -30 to $-40 \mathrm{mV}$ when in the dark. We measured this bias for every data set and subtracted the negative value from our measured voltage. Note that this bias correction lowers our reported visibilities.

\section{Received 23 April 2008; accepted 1 September 2008; published 12 October 2008}

References

1. Abbott, B. et al. Upper limits on a stochastic background of gravitational waves. Phys. Rev. Lett. 95, 221101 (2005)

2. Udem, Th., Reichert, J., Holzwarth, R. \& Hänsch, T. W. Optical frequency measurement of the cesium D1 line with a mode-locked laser. Phys. Rev. Lett. 82, 3568-3571 (1999).

3. Harihan, P. \& Sanders, B. C. Quantum phenomena in optical interferometry. Prog. Opt. 36, 49-128 (1996).

4. Giovannetti, V., Lloyd, S. \& Maccone, L. Quantum-enhanced measurements: Beating the standard quantum limit. Science 306, 1330-1336 (2004).

5. Franson, J. D. Bell inequality for position and time. Phys. Rev. Lett. 62, 2205-2208 (1989).

6. Franson, J. D. Nonlocal cancellation of dispersion. Phys. Rev. A 45, 3126-3132 (1992)

7. Hong, C. K., Ou, Z. Y. \& Mandel, L. Measurement of subpicosecond time intervals between 2 photons by interference. Phys. Rev. Lett. 59, 2044-2046 (1987).

8. Walther, P. et al. de Broglie wavelength of a non-local four-photon state. Nature 429, 158-161 (2004).

9. Mitchell, M. W., Lundeen, J. S. \& Steinberg, A. M. Super-resolving phase measurements with a multiphoton entangled state. Nature 429, 161-164 (2004).

10. Resch, K. J. et al. Time-reversal and super-resolving phase measurements. Phys. Rev. Lett. 98, 223601 (2007).

11. Steinberg, A. M., Kwiat, P. G. \& Chiao, R. Y. Dispersion cancellation in a measurement of the single-photon propagation velocity in glass. Phys. Rev. Lett. 68, 2421-2424 (1992).

12. Larchuk, T. S., Teich, M. C. \& Saleh, B. E. A. Nonlocal cancellation of dispersive broadening in Mach-Zehnder interferometers. Phys. Rev. A 52, 4145-4154 (1995).

13. Bennink, R. S., Bentley, S. J. \& Boyd, R. W. 'Two-photon' coincidence imaging with a classical source. Phys. Rev. Lett. 89, 113601 (2002).

14. Ferri, F. et al. High-resolution ghost image and ghost diffraction experiments with thermal light. Phys. Rev. Lett. 94, 183602 (2005).

15. Bouwmeester, D. et al. Experimental quantum teleportation. Nature 390, 575-579 (1997).

16. Knill, E., Laflamme, R. \& Milburn, G. J. A scheme for efficient quantum computation with linear optics. Nature 409, 46-52 (2001).

17. Steinberg, A. M., Kwiat, P. G. \& Chiao, R. Y. Measurement of the single-photon tunneling time Phys. Rev. Lett. 71, 708-711 (1993).

18. Abouraddy, A. F., Nasr, M. B., Saleh, B. E. A., Sergienko, A. V. \& Teich, M. C. Quantum-optical coherence tomography with dispersion cancellation. Phys. Rev. A 65, 053817 (2002).

19. Nasr, M. B., Saleh, B. E. A., Sergienko, A. V. \& Teich, M. C. Demonstration of dispersion-canceled quantum-optical coherence tomography. Phys. Rev. Lett. 91, 083601 (2003).

20. Fujimoto, J. G. et al. Optical biopsy and imaging using optical coherence tomography. Nature Med. 1, 970-972 (1995)

21. Erkmen, B. I. \& Shapiro, J. H. Phase-conjugate optical coherence tomography. Phys. Rev. A 74 041601 (2006).

22. Banaszek, K., Radunsky, A. S. \& Walmsley, I. A. Blind dispersion compensation for optical coherence tomography. Opt. Commun. 269, 152-155 (2007).

23. Resch, K. J., Puvanathasan, P., Lundeen, J. S., Mitchell, M. W. \& Bizheva, K. Classical dispersion-cancellation interferometry. Opt. Express 15, 8797-8804 (2007).

24. Pe'er, A., Bromberg, Y., Dayan, B., Silberberg, Y. \& Friesem, A. A. Broadband sum-frequency generation as an efficient two-photon detector for optical tomography. Opt. Express 15, 8760-8769 (2007)

25. Karczmarek, J., Wright, J., Corkum, P. \& Ivanov, M. Optical centrifuge for molecules. Phys. Rev. Lett. 82, 3420-3423 (1999). 
26. Xia, J. F., Sanderson, J. H., Liu, W.-K. \& Strickland, D. Experimental observation of Raman chirped adiabatic rapid passage. J. Phys. B 36, L409-L414 (2003).

27. Pessot, M., Maine, P. \& Mourou, G. 1000 times expansion/compression of optical pulses for chirped pulse amplification. Opt. Commun. 62, 419-421 (1987).

28. Paul, H. Interference between independent photons. Rev. Mod. Phys. 58, 209-231 (1986).

29. Altepeter, J., Jeffrey, E. \& Kwiat, P. Phase-compensated ultra-bright source of entangled photons. Opt. Express 13, 8951-8959 (2005).

30. Carrasco, S. et al. Broadband light generation by noncollinear parametric downconversion. Opt. Lett. 31, 253-255 (2006).

31. Drexler, W. Ultrahigh resolution optical coherence tomography. J. Biomed. Opt. 9, 47-74 (2004).

\section{Acknowledgements}

We thank D. Strickland for sharing expertise on pulse compression techniques and K. Bizheva and

G. Weihs for discussions and loaning equipment. We thank J. Sanderson, J. Lundeen, M. Mitchell,

J. Gambetta, A. White and A. Steinberg for comments. This work was supported by NSERC and

CFI. D.B. acknowledges financial support from the Mike and Ophelia Lazaridis Fellowship; R.K. acknowledges financial support from IQC and ORDCF.

Author information

Reprints and permissions information is available online at http://npg.nature.com/reprintsandpermissions. Correspondence and requests for materials should be addressed to R.K. or K.J.R. 